\title{
Adding Ozone to Dextrose and Somatropin for Intra-articular Knee Prolotherapy: A Randomized Single-Blinded Controlled Trial
}

\author{
Farnad Imani $\mathbb{D}^{1}{ }^{1}$, Kokab Hejazian ${ }^{1}$, Mohammad-Reza Kazemi ${ }^{1}$, Mahnaz Narimani-Zamanabadi ${ }^{2,{ }^{*}}$ \\ and Khalid M Malik ${ }^{3}$ \\ ${ }^{1}$ Pain Research Center, Department of Anesthesiology and Pain Medicine, Iran University of Medical Sciences, Tehran, Iran \\ ${ }^{2}$ Department of Anesthesiology, Tehran Medical Science, Islamic Azad University, Tehran, Iran \\ ${ }^{3}$ Division of Pain Medicine, Department of Anesthesiology, College of Medicine, University of Illinois, Chicago, USA \\ "Corresponding author: Department of Anesthesiology, Islamic Azad University, Tehran Medical Sciences Branch, Tehran, Iran. Email: mahnaznarimani@gmail.com
}

Received 2020 October 18; Accepted 2020 October 22.

\begin{abstract}
Background: Prolotherapy, as an alternative therapy, has emerged as an effective treatment for chronic musculoskeletal injury, including knee osteoarthritis (OA). Several studies have mention ozone as a potential treatment for these diseases, which is based on analgesic, anti-inflammatory, and anti-oxidant.

Objectives: The current study aimed to investigate the effect of adding ozone gas to hypertonic dextrose and somatropin for knee prolotherapy in patients with knee OA. For this purpose, pain, knee stiffness, and physical activity are measured.

Methods: Sixty patients with chronic knee OA were randomly assigned into two groups of DS and DSO. The DS group received intraarticular hypertonic dextrose $(10 \mathrm{ml}$ ) plus $4 \mathrm{IU}$ somatropin (4 IU), and the DSO group received $10 \mathrm{ml}$ ozone 25 mcg plus intervention in the DS group. This procedure was performed three times (first, third, and fifth weeks). WOMAC score was examined during the third, fifth, and sixteenth weeks.

Results: The mean WOMAC score of the DS group was decreased significantly $(\mathrm{P}<0.001)$ sixteen weeks after providing the intervention (before $64.9 \pm 10.6$, vs. after $49.2 \pm 9.0$ ). A similar decrease $(\mathrm{P}<0.001$ ) was observed in the DSO group (before $64.1 \pm 11.3$, vs. after $41.3 \pm 8.0$ ). The decrease of the WOMAC score in the third and sixteenth weeks after providing the intervention was significant in the DSO group compared to the DS group $(\mathrm{P}<0.005)$.

Conclusions: For patients with knee OA, prolotherapy with ozone plus hypertonic dextrose and somatropin was more effective in sedating the pain and improving the stiffness and function of the knee than dextrose and somatropin alone.
\end{abstract}

Keywords: Knee, Osteoarthritis, Prolotherapy, Dextrose, Ozone, Somatropin

\section{Background}

Osteoarthritis (OA) is a major cause of pain, stiffness, and malfunctioning of joints, which carries considerable disability in adults (1). OA most commonly affects the knees because of the mechanical pressure being applied in the weight-bearing process (2). The goals of OA treatment are to sedate the pain, reducing swelling and disability, and improving the patients' quality of life. Few effective treatments are available for those who suffer from OA, including pharmacologic (analgesics, nonsteroidal anti-inflammatory) and non-pharmacologic interventions (muscle strengthening, physical and herbal therapies, intra-articular injection, arthroscopic, and arthroplasty surgery) (3). To date, however, no pharmacological intervention could stop the progressive nature of OA. A recent systematic review has shown that intra-articular in- jections are preferable to nonsteroidal anti-inflammatory drugs in the treatment of OA (4).

Prolotherapy, a well-known proliferative therapy, is a therapeutic option beneficial for musculoskeletal disorders such as tendonitis and OA in recent years (5). Although it has been used for several decades to treat knee $\mathrm{OA}$, but its mechanism of action is not clear yet. Solutions such as hyperosmolar dextrose and platelet-rich plasma are serially injected as regenerative solutions to stimulate, theoretically, host inflammatory response that, in turn, strengthens connective tissue, especially collagen fibers, and improves biomechanics and joint function $(6,7)$. The application of prolotherapy and its efficacy is a matter of controversy. Besides, evidence generated by experimental and clinical studies are not sufficient to make a decision (8).

Ozone is another potential therapeutic option in ad- 
dition to pharmacological and rehabilitation therapies. Recent experimental studies proposed that the mechanism of action of ozone is based on analgesic, antiinflammatory, and anti-oxidant properties. It's about a decade that ozone is using to treat several musculoskeletal disorders. Moreover, there are evidence of its effectiveness in treating chronic low back pain $(9,10)$.

The current study aimed to assess the efficacy of adding ozone to hypertonic dextrose and somatropin for intraarticular knee prolotherapy in the OA patients by considering pain, stiffness, and physical activity of the knee as the main outcomes.

\section{Objectives}

The current study aimed to assess the efficacy of adding ozone to hypertonic dextrose and somatropin for intraarticular knee prolotherapy in the OA patients by considering pain, stiffness, and physical activity of the knee as the main outcomes.

\section{Methods}

After obtaining approval of the Ethical Committee and written informed consent, 60 eligible participants with chronic knee OA (diagnosed based on the criteria of American College of Rheumatology) and a knee radiologic stage 1-3 (based on the Kellgren-Lawrence criteria) were enrolled in this randomized single-blinded controlled trial study. The study design and protocol were registered at the Iranian Registry of Clinical Trials (IRCT201410187984N20).

Inclusion criteria were being aged 50 - 75 years, ASA I-II, knee pain for at least three months or longer, and no intra-articular injections. Exclusion criteria were drug abuse, hemophilia, diabetes, anticoagulation therapy, history of total knee replacement, inflammatory or septic knee arthritis, allergy or intolerance to medication, and knee trauma.

Participants were randomly separated into the groups of DS (hypertonic dextrose and somatropin $(n=30)$ ) or DSO (hypertonic dextrose, somatropin, and ozone $(n=30)$ ).

\subsection{Intervention}

After intravenous cannula insertion, standard monitoring (ECG, HR, and pulse oximetry), on supine position, and aseptic conditions, patients were prepared for the intervention. In both groups, local anesthesia was conducted with $2 \mathrm{ml}$ lidocaine $1 \%$, by using a 22 -gauge needle by the pain physician, from the superolateral part of the patella with an angle of about $45^{\circ}$ under the fluoroscopic guidance which entered the knee joint space. Afterward, confirmation was performed by articular fluid aspiration. In the DS group, $10 \mathrm{ml}$ of 50\% hypertonic dextrose and 4 IU human somatropin (Eutropin, LG, Korea) was injected. In the DSO group, in addition to $10 \mathrm{ml}$ of $50 \%$ hypertonic dextrose and $4 \mathrm{IU}$ somatropin, $10 \mathrm{ml}$ of ozone $25 \mathrm{mcg}$ was added. Following the intervention, patients were monitored for potential side effects such as pain, swelling, and hemorrhage. Each patient received three intra-articular knee injections in weeks zero, three, and five. After providing all three procedures, patients were assessed by another researcher, who was local anesthesia, at the third, fifth, and sixteenth weeks, and the WOMAC score was calculated. Participants were asked not to use any medications, particularly NSAIDs, after initiation of prolotherapy and during the study period. In the case of intolerance pain, they were ordered to use oral acetaminophen $500 \mathrm{mg}$ up to three times a day.

\subsection{Outcome Measures}

The primary outcome was variations in the kneerelated quality of life [measured by the Western Ontario McMaster University Osteoarthritis Index (WOMAC)]. The WOMAC questionnaire contains three subscales: (1) pain (5 items); (2) stiffness (2 items); and (3) physical function (17 items). The subscales are scored on a five-point Likert scale, ranging from zero (nothing) to four (extreme), with the total score ranging from zero (lowest) to 96 (highest).

\subsection{Statistical Analysis}

Data were analyzed using SPSS version 18 (IBM, USA). The chi-square and independent t-tests were used to analyze continuous and categorical variables, respectively. Descriptive statistics and outcomes at each time point were described by the mean and standard deviation (SD). Repeated measures analysis was used to compare WOMAC total and subscale scores at different times. Statistical significance was considered when P-value $<0.05$.

\section{Results}

A total of 60 patients were investigated in the present study. The overall characteristics of the participants are provided in Table 1. After Sixteen weeks, the mean WOMAC score in the DS group was decreased significantly from 64.9 \pm 10.6 (before the procedure) to $49.2 \pm 9.0$ (after the procedure $)(\mathrm{P}<0.001)$. A similar decrease was observed for the DSO group, so that in the sixteenth week after providing the intervention, the mean WOMAC score was decreased significantly (from $64.1 \pm 11.3$ to $41.3 \pm 8.0$ ( $\mathrm{P}<0.001)$ ). The observed decrease in WOMAC score in the third and sixteenth weeks after providing the intervention was significantly lower in the DSO group compared to the DS group 
$(\mathrm{P}<0.005)$. We have also compared the WOMAC scores of both groups for different subscales at the third, fifth, and sixteenth weeks after providing the treatment. Subjects of the DSO group had lower pain and stiffness score in the third week (Figure 2 and 3). Also, the function score was lower in these patients at the third, fifth, and sixteenth weeks compared to those in the DS group $(\mathrm{P}<0.05)$ (Figure 4).

\begin{tabular}{|c|c|c|c|}
\hline Variables & $\operatorname{DS}(\mathbf{n}=\mathbf{3 0})$ & $\operatorname{DSO}(\mathbf{n}=30)$ & PValue \\
\hline Age, $y$ & $65.5 \pm 6.4$ & $67.7 \pm 8.2$ & 0.257 \\
\hline Gender & & & 0.922 \\
\hline Male & $11(36.6)$ & $10(33.3)$ & \\
\hline Female & $19(63.3)$ & $20(66.6)$ & \\
\hline OA severity & & & 0.854 \\
\hline Grade 1 & $3(10.0)$ & $2(6.7)$ & \\
\hline Grade 2 & $10(33.3)$ & $11(36.6)$ & \\
\hline Grade 3 & $17(56.7)$ & $17(56.7)$ & \\
\hline WOMAC score & $64.9 \pm 10.6$ & $64.1 \pm 11.3$ & 0.806 \\
\hline
\end{tabular}

${ }^{\mathrm{a}}$ Values are expressed as No. (\%) or mean \pm SD.

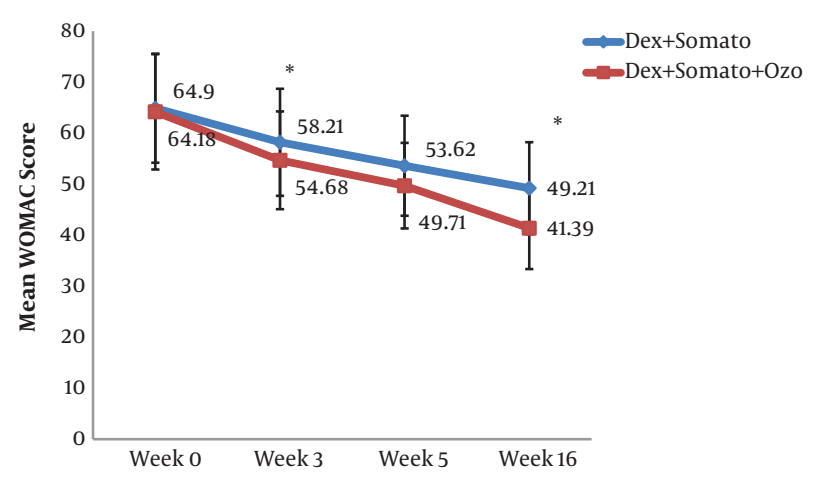

Figure 1. Mean WOMAC score of patients at three, five, and sixteen weeks after treatment. * P-value $<0.005$.

\section{Discussion}

The main objective of the present study was to investigate the efficacy of adding ozone gas to hypertonic dextrose and Somatropin for knee prolotherapy in OA patients to sedate their pain and to improve stiffness and function of the knee, measured by the WOMAC score. Based on the findings, participants of both groups experienced substantial improvements in all dimensions of the WOMAC during the study period. However, the observed improvement

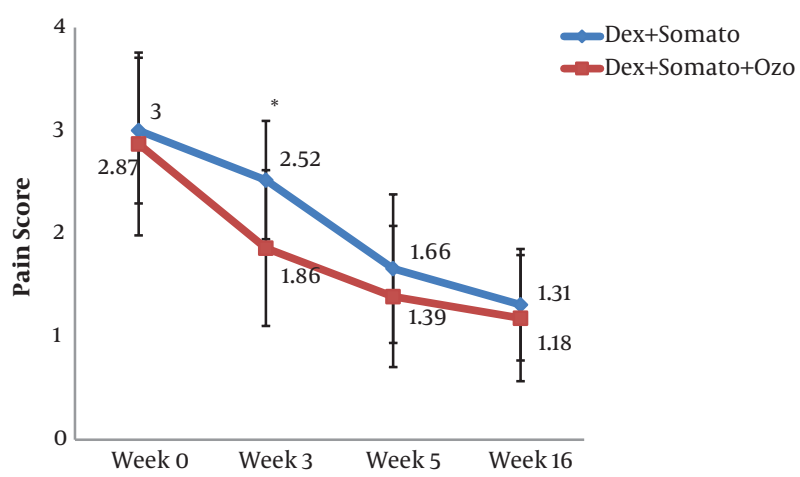

Figure 2. Mean pain score of patients at third, fifth, and sixteenth weeks after treatment. * P-value $<0.005$

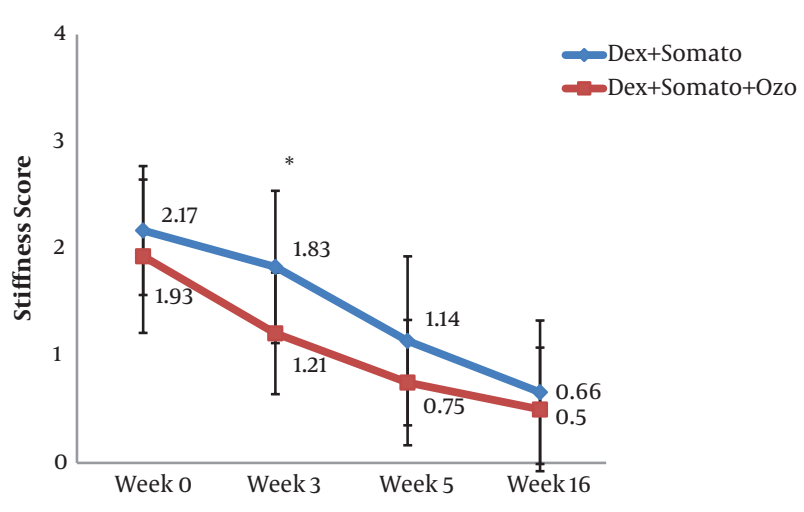

Figure 3. Mean stiffness score of patients at third, fifth, and sixteenth weeks after treatment. ${ }^{*}$ P-value $<0.005$.

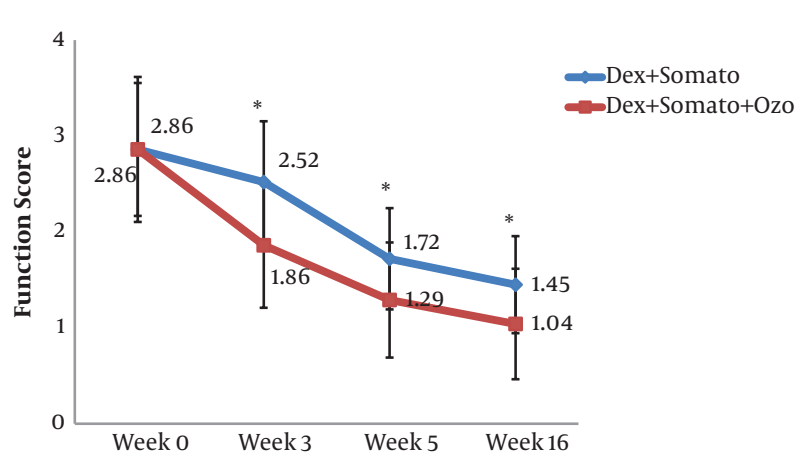

Figure 4. Mean function score of at third, fifth, and sixteenth weeks after treatment. ${ }^{*}$ P-value $<0.005$.

was higher in the group that received ozone in addition to dextrose and somatropin. In a single armed clinical study conducted by Eslamian et al., prolotherapy with dextrose could significantly decrease patients' pain both at 
rest and during activity. Also, the authors reported that the intervention improved range of motion (ROM) in the knee joint and WOMAC scores during their six months follow up. Also, Rabbago et al., in a study with 52 weeks of follow-up, reported a significant decrease in WOMAC score, pain, stiffness, and function (11). Previous studies with various follow-up periods have assumed that because of the increased amount of cartilage volume after prolotherapy sessions, it would result in significant improvement in the WOMAC score in a short period, particularly regarding the pain subscale, and this effect will decrease over time. The observed similarities between the findings of studies with long-term follow-ups can be attributed to this issue (12-14). In accordance with our study, most of the investigations in this field have considered the intra-articular injections by targeting the damaged cartilage and intra-articular ligaments.

There are studies that utilized dextrose for knee prolotherapy (both intra and extra-articular injections) and have reported promising results concerning pain relief, stiffness, and increased function in OA patients (15). In a clinical study on the effect of prolotherapy with erythropoietin in comparison to dextrose, Rahimzadeh et al. reported that erythropoietin, compared to hypertonic dextrose, had more efficacy in reducing pain and affecting the range of motion.

In the present study, by adding ozone to the composition of intra-articular injection with dextrose and somatropin, the WOMAC score of participants was significantly improved compared to the control group. It seems that ozone has augmented the possible mechanism by which prolotherapy affects patients with knee OA. The composition of dextrose, somatropin, and ozone activated different biological aspects of the healing process in joints. To date, experimental studies have mentioned the role of inflammation, soft tissue size, and strength for the effect of prolotherapy with dextrose (16). Prolotherapy injections stimulated an inflammatory response, which in turn led to secondary production of growth factors without causing any damage and initiation of proliferation phase in the joint (17). Erythropoietin also plays a pivotal role in this composition. Recent animal trials have revealed that erythropoietin stimulated osteogenesis, angiogenesis, and proliferation in a femoral segmental defect (18). Besides, Mihmanli et al. evaluated the efficacy of subcutaneous administration of recombinant human erythropoietin in osteogenesis and concluded that it could improve the rate and quality of bone healing during distraction osteogenesis (19).

On the other hand, multiple mechanisms are mentioned as possible causes of the ozone's effect on joints. It promotes angiogenesis at the level of cartilaginous by triggering local micro-vascularization and modulates the cytokines during the inflammation process by antiinflammatory role $(20,21)$. Also, it has been argued that ozone therapy can promote wound healing by the proliferation of VEGF and TGF- $\alpha$ at the early stages of the treatment (22). Intra-articular knee ozone injection can sedate the pain, similar to prolotherapy, by hypertonic dextrose (23). Also, Hashemi et al. have reported the significant efficacy of ozone (intradiscal injection) on pain relief and disability improvement in patients with low back pain caused by disc prolapsed (24). In contrast to their study, we designed a RCT on specifically knee OA and evaluated the outcome by a valid scale that contains three aspects of healthrelated quality of the knee joint and found significant results, particularly in short-term follow-up.

The present study had some limitations, including a small sample size and using self-reporting to collect information. Therefore, the authors recommend performing high-quality clinical trials with a larger sample size. Also, comparing the efficacy of prolotherapy with other complementary therapeutic options could be a matter of future research. Our encouraging finding for ozone could be a promising start for further investigations to clarify the effects of ozone therapy and its possible combinations.

In conclusion, according to the findings, for patients with knee OA, prolotherapy with ozone plus hypertonic dextrose and somatropin was more effective in sedating the pain and improving stiffness and function of the knee than dextrose and somatropin alone.

\section{Footnotes}

Authors' Contribution: Study concept and design: FI; acquisition of data: $\mathrm{KH}$ and MRK; data analysis and interpretation: KH and FI; drafting of the manuscript: KH and MNZ; Critical revision of the manuscript for important intellectual content: FI and KMM; study supervision: FI.

Clinical Trial Registration Code: IRCT201410187984N20. Conflict of Interests: The authors declare no conflict of interest.

Ethical Approval: The ethics committee reference number is 24672-30-02-93.

Funding/Support: None declared.

Informed Consent: All patients signed informed consent forms before entering the study.

\section{References}

1. Felson DT. Clinical practice. Osteoarthritis of the knee. N Engl J Med. 2006;354(8):841-8. doi: 10.1056/NEJMcp051726. [PubMed: 16495396].

2. Felson DT. The sources of pain in knee osteoarthritis. Curr Opin Rheumatol. 2005;17(5):624-8. doi: 10.1097/01.bor.0000172800.49120.97. [PubMed:16093843]. 
3. Imani F, Patel VB. Therapeutic challenges for knee osteoarthritis. Anesth Pain Med. 2019;9(3). e95377. doi: 10.5812/aapm.95377.

4. Bannuru RR, Schmid CH, Kent DM, Vaysbrot EE, Wong JB, McAlindon TE. Comparative effectiveness of pharmacologic interventions for knee osteoarthritis: a systematic review and network meta-analysis. Ann Intern Med. 2015;162(1):46-54. doi: 10.7326/M14-1231. [PubMed: 25560713].

5. Reeves KD, Hassanein KM. Long-term effects of dextrose prolotherapy for anterior cruciate ligament laxity. Altern Ther Health Med. 2003;9(3):58-62. [PubMed: 12776476].

6. Rahimzadeh P, Imani F, Faiz SHR, Entezary SR, Zamanabadi MN, Alebouyeh MR. The effects of injecting intra-articular platelet-rich plasma or prolotherapy on pain score and function in knee osteoarthritis. Clin Interv Aging. 2018;13:73-9. doi: 10.2147/CIA.S147757. [PubMed: 29379278]. [PubMed Central: PMC5757490].

7. Solmaz I, Deniz S, Cifci OT. Treatment of advanced stage gonarthrosis with prolotherapy: case report. Anesth Pain Med. 2014;4(1) e9171. doi: 10.5812/aapm.9171. [PubMed: 24660149]. [PubMed Central: PMC3961030].

8. Tieppo Francio V, Dima RS, Towery C, Davani S. Prolotherapy and Low Level Laser Therapy: A Synergistic Approach to Pain Management in Chronic Osteoarthritis. Anesth Pain Med. 2017;7(5). e14470. doi: 10.5812/aapm.14470. [PubMed: 29696113]. [PubMed Central: PMC5903214].

9. Magalhaes FN, Dotta L, Sasse A, Teixera MJ, Fonoff ET. Ozone therapy as a treatment for low back pain secondary to herniated disc: a systematic review and meta-analysis of randomized controlled trials. Pain Physician. 2012;15(2):E115-29. [PubMed: 22430658].

10. Rahimzadeh P, Imani F, Ghahremani M, Faiz SHR. Comparison of percutaneous intradiscal ozone injection with laser disc decompression in discogenic low back pain. J Pain Res. 2018;11:1405-10. doi: 10.2147/JPR.S164335. [PubMed: 30104895]. [PubMed Central: PMC6074825].

11. Rabago D, Zgierska A, Fortney L, Kijowski R, Mundt M, Ryan M, et al. Hypertonic dextrose injections (prolotherapy) for knee osteoarthritis: results of a single-arm uncontrolled study with 1-year follow-up. J Altern Complement Med. 2012;18(4):408-14. doi: 10.1089/acm.2011.0030. [PubMed: 22515800]. [PubMed Central: PMC3326267].

12. Rahimzadeh P, Imani F, Faiz SH, Alebouyeh MR, Azad-Ehyaei D, Bahari L, et al. Adding intra-articular growth hormone to platelet rich plasma under ultrasound guidance in knee osteoarthritis: A comparative double-blind clinical trial. Anesth Pain Med. 2016;6(6). e41719. doi: 10.5812/aapm.41719. [PubMed: 28975078]. [PubMed Central: PMC5560632]

13. Eslamian F, Amouzandeh B. Therapeutic effects of prolotherapy with intra-articular dextrose injection in patients with moderate knee osteoarthritis: a single-arm study with 6 months follow up. Ther Adv Musculoskelet Dis. 2015;7(2):35-44. doi: 10.1177/1759720X14566618. [PubMed: 25829954]. [PubMed Central: PMC4357593].

14. Imani F, Entezary S, Razi M, Jafarian AA, Yousefshahi F, Etemadi H, et al. The effect of intra-articular meperidine and bupivacaine $0.5 \%$ on postoperative pain of arthroscopic knee surgery; a randomized double blind clinical trial. Anesth Pain Med. 2015;5(1). e27470. doi: 10.5812/aapm.27470.

15. Rabago D, Patterson JJ, Mundt M, Kijowski R, Grettie J, Segal NA, et al. Dextrose prolotherapy for knee osteoarthritis: a randomized controlled trial. Ann Fam Med. 2013;11(3):229-37. doi: 10.1370/afm.1504. [PubMed: 23690322]. [PubMed Central: PMC3659139].

16. Rahimzadeh P, Imani F, Faiz SH, Entezary SR, Nasiri AA, Ziaeefard M. Investigation the efficacy of intra-articular prolotherapy with erythropoietin and dextrose and intra-articular pulsed radiofrequency on pain level reduction and range of motion improvement in primary osteoarthritis of knee. J Res Med Sci. 2014;19(8):696-702. [PubMed: 25422652]. [PubMed Central: PMC4235087].

17. Jensen KT, Rabago DP, Best TM, Patterson JJ, Vanderby RJ. Early inflammatory response of knee ligaments to prolotherapy in a rat model. J Orthop Res. 2008;26(6):816-23. doi: 10.1002/jor.20600. [PubMed: 18240327]. [PubMed Central: PMC2755507].

18. Holstein JH, Orth M, Scheuer C, Tami A, Becker SC, Garcia P, et al. Erythropoietin stimulates bone formation, cell proliferation, and angiogenesis in a femoral segmental defect model in mice. Bone. 2011;49(5):1037-45. doi: 10.1016/j.bone.2011.08.004. [PubMed: 21851867].

19. Mihmanli A, Dolanmaz D, Avunduk MC, Erdemli E. Effects of recombinant human erythropoietin on mandibular distraction osteogenesis. J Oral Maxillofac Surg. 2009;67(11):2337-43. doi: 10.1016/j.joms.2008.06.082. [PubMed:19837300].

20. Chen $\mathrm{H}, \mathrm{Yu} \mathrm{B}, \mathrm{Lu} \mathrm{C}$, Lin Q. The effect of intra-articular injection of different concentrations of ozone on the level of TNF-alpha, TNF-R1, and TNF-R2 in rats with rheumatoid arthritis. Rheumatol Int. 2013;33(5):1223-7. doi: 10.1007/s00296-012-2529-7. [PubMed: 23052485].

21. Vaillant JD, Fraga A, Diaz MT, Mallok A, Viebahn-Hansler R, Fahmy Z, et al. Ozone oxidative postconditioning ameliorates joint damage and decreases pro-inflammatory cytokine levels and oxidative stress in PG/PS-induced arthritis in rats. Eur J Pharmacol. 2013;714(1-3):318-24. doi: 10.1016/j.ejphar.2013.07.034. [PubMed: 23911887].

22. Zhang J, Guan M, Xie C, Luo X, Zhang Q, Xue Y. Increased growth factors play a role in wound healing promoted by noninvasive oxygen-ozone therapy in diabetic patients with foot ulcers. Oxid Med Cell Longev. 2014;2014:273475. doi: 10.1155/2014/273475. [PubMed: 25089169]. [PubMed Central: PMC4096389].

23. Hashemi M, Jalili P, Mennati S, Koosha A, Rohanifar R, Madadi F, et al. The effects of prolotherapy with hypertonic dextrose versus prolozone (intraarticular ozone) in patients with knee osteoarthritis. Anesth Pain Med. 2015;5(5). e27585. doi: 10.5812/aapm.27585. [PubMed: 26587401]. [PubMed Central: PMC4644302].

24. Hashemi M, Poorfarokh M, Mohajerani SA, Jalili P, Akhyani V, Barikani A, et al. Injection of intradiscal 02-03 to reduce pain and disability of patients with low back pain due to prolapsed lumbar disk. Anesth Pain Med. 2014;4(5). e19206. doi: 10.5812/aapm.19206. [PubMed: 25798376]. [PubMed Central: PMC4358330]. 